\title{
Transferable, Polarizable Force Field for Electrolytes, Protic Ionic Liquids and Deep Eutectic Solvents
}

\author{
Kateryna Goloviznina, Zheng Gong, Margarida Costa Gomes, and Agílio A. H. Páduaa) \\ Laboratoire de Chimie, École Normale Supérieure de Lyon \& CNRS, 69364 Lyon, \\ France
}

(Dated: 13 November 2020)

The transferable, polarizable CL\&Pol force field for aprotic ionic liquids presented in our previous study (J. Chem. Theory Comput. 2019, 15, 5858, DOI: 10.1021/acs.jctc.9b00689) is extended to electrolytes, protic ionic liquids, deep eutectic solvents, and glycols. These systems are problematic in polarizable simulations because they contain either small, highly charged ions or strong hydrogen bonds, which cause trajectory instabilities due to the pull exerted on the induced dipoles. We use a Tang-Toennies function to dampen, or smear, the interactions between charges and induced dipole at short range involving small, highly charged atoms (such as hydrogen or lithium), thus preventing the "polarization catastrophe". The new force field gives stable trajectories and is validated through comparison with experimental data on density, viscosity, and ion diffusion coefficients of liquid systems of the above-mentioned classes. The results also shed light on the hydrogen-bonding pattern in ethylammonium nitrate, a protic ionic liquid, for which the literature contains conflicting views. We describe the implementation of the Tang-Toennies damping function, of the temperature-grouped Nosé-Hoover thermostat for polarizable molecular dynamics and of the periodic perturbation method for viscosity evaluation from non-equilibrium trajectories in the LAMMPS molecular dynamics code. The main result of this work is the wider applicability of the CL\&Pol polarizable force field to new, important classes of fluids, achieving robust trajectories and a good description of equilibrium and transport properties in challenging systems. The transferability and fragment-based approach of CL\&Pol will allow ready extension to a wide variety of protic ionic liquids, deep eutectic solvents and electrolytes.

\section{INTRODUCTION}

Molecular force fields that include polarization explicitly bring significant improvements to the representation structure, energetics and dynamics of molecular and ionic liquids. In some systems, however, strong electrostatic interactions may cause instability problems in the induced dipoles that represent induction (or polarization) effects, resulting in a so-called "polarization catastrophe". Examples of problematic systems include electrolytes containing small ions such as $\mathrm{Li}^{+}$, protic ionic liquids (PIL) such as ethylammonium nitrate (EAN), deepeutectic solvents (DES) that have strong hydrogen bonds between a $\mathrm{H}$-bond donor and an organic salt, and also glycols, which contain strong, persistent intramolecular H-bonds.

Each of these classes of system is interesting in its own right for fundamental and applied research. Protic ionic liquids are promising for energy storage and catalytic organic synthesis, ${ }^{1,2}$ exploiting the proton transfer and the solvation properties of ionic media. Ethylammonium nitrate, one of the prominent PIL, has a $3 \mathrm{D}$ hydrogen-bond network similar to water, ${ }^{3}$ and is actively studied since its micro-structure is still object of debate. ${ }^{4-11}$ Deep eutectic solvents, unlike ionic liquids, are not completely ionic, since they are formed by a salt mixed with a molecular compound, namely a hydrogenbond donor: urea, glycol or carboxylic acid being among

a) Electronic mail: agilio.padua@ens-lyon.fr the most common examples. By changing the the ratio between the components or substitution of one molecular compound by another, the properties of these systems can be easily tuned, broadening their applications in electrodeposition of metals, biomass transformation, gas capture and organic synthesis. ${ }^{12,13}$ Solutions of alkali metal cations (lithium, sodium) in ionic liquids produces safe, non-flammable electrolytes that offer new perspectives for designing batteries of increased their capacity and charge-discharge timescales. ${ }^{14-17}$

Several techniques can be used to incorporate polarization explicitly in force fields, such as point induced dipoles or Drude induced dipoles, as well as fluctuating charges, but all may suffer from instability issues, ${ }^{18-24}$ especially in systems with strong electrostatic interactions, so building a robust polarizable force field is challenging.

Here we extend the applicability of our force polarizable force field for ionic liquids, CL\&Pol, ${ }^{25,26}$ which can describe a wide variety of aprotic ionic liquids and their mixtures with molecular compounds, and include polarization via Drude induced dipoles placed on atomic sites. These Drude induced dipoles are formed by a pair of partial charges of opposite sign, the Drude core (DC, usually positive) and the Drude particle (DP, usually negative), bound by a harmonic spring. The equilibrium elongation of the spring is zero (each DC does not interact with its own DP) so the induced dipole moment is zero in the absence of an external electric field, but reacts to the electrostatic environment. The values of the partial charge and spring force constant are determined by the atomic polarizability of the atom, so these parameters have physical meaning. Drude induced dipoles have been 
used successfully in polarizable force fields. ${ }^{27,28}$ One important aspect of the CL\&Pol force field is the fragmentbased transferability inherited from the non-polarizable CL\&P force field ${ }^{29-31}$ that allows the model to describe broad families of cation and anions with different functional groups, represented by smaller building blocks, or "fragments", and no specific parameterization is required for each individual compound. Of course, parameters for new fragments have to be calculated following a general procedure. We extend the CL\&Pol model to the abovementioned systems, which contain densely-charged ions or strong H-bonds involving ions. The dominating interactions in DES and PIL, or in electrolytes with denselycharged ions, are Coulomb forces and H-bonds involving ions and it is the importance of the latter that made the extension of the CL\&Pol force to DES and PIL challenging.

Strong H-bonds are established for example between $\mathrm{H}$ atoms bound to $\mathrm{N}$ or $\mathrm{O}$ (the $\mathrm{H}$-bond donor) and negatively charged atoms (the acceptor). But in the OPLSAA force field the $\mathrm{H}$ atoms are represented by point charges only, embedded in the Lennard-Jones sites of the heavier atoms, about $1 \AA$ from their center. Glycols are molecular compounds that prove challenging for polarizable simulations because of the intramolecular H-bonds involving $\mathrm{OH}$ groups, which may persist for a long time. The absence of repulsive core around these $\mathrm{H}$ atoms and the strong electrostatic pull on Drude dipoles belonging to the H-bond acceptor may lead to unstable trajectories, a so-called "polarization catastrophe", which the thermostat handling the Drude degrees of freedom cannot correct.

Another source of "polarization catastrophe" may be an excessive correlation between nearby induced dipoles. This can be avoided by damping inter- and intramolecular dipole-dipole interactions with a Thole damping function ${ }^{19,32,33}$ that represents smearing of the electron cloud. This device has become a standard for polarizable simulations and is implemented in the major molecular dynamics packages, but we found it inefficient to handle strong hydrogen-bonded systems.

Alternative strategies to correct unstable trajectories are based either on preventing a DP from leaving its DC (a hard or reflecting wall) or applying a restoring force when a significant displacement occurs. Thus, Roux et l. $^{23}$ suggested introducing an additional anharmonic force into the systems containing highlypolarizable halide anions that is activated only beyond a certain DC-DP distance so that the linear polarization response is conserved for small DC-DP elongations. Later, these authors proposed using a "hard wall" 24 to limit the maximum displacement of the DP from its DC: when a DP reaches a user-defined limit, typically of $0.2-$ $0.25 \AA$, it is reflected back towards the parent site along the DP-DC bond by scaling the positions and the velocities of the DC-DP pair in question (this is implemented in GROMACS ${ }^{34}$ and OpenMM ${ }^{35}$ ). Yet other solutions are to associate a small repulsive core to the Drude particle, as is done in the CHARMM force field, ${ }^{36}$ or to introduce an electric field-dependent polarizability to account for non-linear polarization effects. ${ }^{37}$

The most promising way to prevent instabilities in strong H-bonded systems is to add a stiff distancedependent damping potential that keeps charge-dipole interactions finite at short distances and can be used in conjunction with the Thole function. For this purpose, we adapted the Tang-Toennies (TT) damping function, ${ }^{38}$ which although developed originally for short-range damping of dispersion interactions, has been adapted for use in polarizable simulations of molten salts. ${ }^{39-43}$

The main focus of the present work is the implementation and validation of specific short-range damping of strong charge-dipole interactions in order to ensure the stability of molecular dynamics trajectories in our problematic systems, and thus allowing the extension of the CL\&Pol force field no important new classes of systems. Otherwise, we followed the strategy of our polarizable force field for ionic liquids, CL\&Pol, which is a polarizable version of the widely-used CL\&P fixed-charge force field for ionic liquids, ${ }^{29-31}$ compatible with OPLS$\mathrm{AA}^{44}$ and therefore opening the possibility of simulating broad classes of molecular compounds, ions and materials. The parameters needed to describe polarization effects in CL\&Pol can be obtained from quantum calculations, either using symmetry-adapted perturbation theory (SAPT), or from a predictive scheme based on accessible molecular quantities such as polarizabilities and electrostatic charge distributions ${ }^{25}$ avoiding costly highlevel calculations.

\section{SIMULATION DETAILS}

\section{A. Molecular dynamics setup}

Molecular dynamics (MD) simulations of periodic cubic boxes containing 10 000-15000 atoms were performed in LAMMPS $^{45}$ with the USER-DRUDE ${ }^{46}$ package enabled. Starting configurations and force field were prepared using the fftool ${ }^{47}$ and Packmol ${ }^{48}$ utilities and then converted to the polarizable force field using the polarizer and scaleLJ tools. ${ }^{49}$ A cut-off of $12 \AA$ was considered for pair potentials with tail corrections applied. Electrostatic energies were evaluated using the particle-particle particle-mesh (PPPM) method with an accuracy of $10^{-5}$.

Regarding simulation conditions, the time step was $1 \mathrm{fs}$ and the pressure was kept at 1 bar, while different temperatures were chosen for different systems according to the availability of experimental data for comparison. After 2 ns equilibrations, 10 ns trajectories of liquid systems were generated in the $N p T$ ensemble using conventional and temperature-grouped ${ }^{50}$ Nosé-Hoover thermostat and barostat. The crystal structure of EAN was modeled for $1 \mathrm{~ns}$ after the relaxation of the box.

The structure factor of liquid systems was calculated 
using the TRAVIS software, ${ }^{51,52}$ with atomic form factors or cross sections corresponding to X-ray and neutron scattering,

$$
\begin{array}{r}
S(q)=\frac{\sum_{i=1}^{N} \sum_{j=1}^{N} x_{i} x_{j} f_{i}(q) f_{j}(q) H_{i j}(q)}{\left(\sum_{i=1}^{N} x_{i} f_{i}(q)\right)^{2}} \\
H_{i j}(q)=4 \pi \rho_{0} \int_{0}^{r_{\max }} r^{2}\left(g_{i j}(r)-1\right) \frac{\sin (q r)}{q r} d r
\end{array}
$$

Dynamic properties were evaluated from the trajectories according to recent recommendations. ${ }^{53}$ The GreenKubo relation was used to evaluate shear viscosity using the off-diagonal components of pressure tensor from the equilibrium trajectory,

$$
\eta=\frac{V}{k T} \int_{0}^{\infty}\left\langle p_{x y}(t) p_{x y}(0)\right\rangle d t
$$

The tail of the autocorrelation function (ACF) of GreenKubo relation was smoothed with the following exponential decay function to eliminate the noise at long times,

$$
S_{\mathrm{ACF}}^{f}(t)=a \exp \left(-t^{\beta}\right)
$$

where $a$ and $\beta$ are obtained from fit to the non-oscillatory decay section of the ACF. ${ }^{25}$ An alternative approach of fitting the "running integral" of the Green-Kubo relation would require averaging over many independent simulations $^{53}$ is computationally more expensive, especially for polarizable simulations. We will use here viscosity values from equilibrium MD to analyse the tuning of force field parameters, but prefer to compute viscosity from non-equilibrium MD for the final force field.

The viscosity from non-equilibrium MD simulations was evaluated using the periodic perturbation method, ${ }^{54}$ by applying a cosine-shaped acceleration along the $x$-axis with periodicity in the $z$-axis,

$$
\frac{1}{\eta}=\left\langle\frac{\mathcal{V}}{\mathcal{A}} \frac{4 \pi^{2}}{l_{z}^{2} \rho}\right\rangle
$$

where $\mathcal{A}$ is the amplitude of the acceleration, $l_{z}$ is the height of the box and $\mathcal{V}$ is the amplitude of the velocity profile generated by the applied acceleration. The choice of the amplitude of the acceleration is discussed in the Appendix.

Einstein's relation was applied to the mean square displacements to calculate diffusion coefficients

$$
D=\lim _{x \rightarrow \infty} \frac{1}{6} \frac{d}{d t}\left\langle(\mathbf{r}(t)-\mathbf{r}(0))^{2}\right\rangle
$$

The Yeh-Hummer correction ${ }^{53,55}$ was used to account for finite-size effects,

$$
D_{0}=D+\frac{2.8373 k_{B} T}{6 \pi \eta L}
$$

where $k_{B}$ is the Boltzmann constant, $L$ is the length of a cubic box, and $\eta$ is the shear viscosity of the system at temperature $T$, evaluated here from non-equilibrium MD simulations since these have smaller uncertainties. We report below some very high viscosity values obtained during the tuning of force field parameters, and we did not apply this correction to those intermediate results. The results with the final force field include the correction.

\section{B. Thermostat for Drude degrees of freedom}

When simulating a polarizable system with Drude induced dipoles in molecular dynamics, a self-consistent iterative procedure should be used to converge the energy at each timestep. This is time-consuming, so a faster method based on the extended Lagrangian approach is commonly employed, where the Drude particles are treated as additional dynamical degrees of freedom (DOF). In order to prevent thermal energy transfer between the real molecular DOF and the Drude DOF, the relative motion of Drude particles with respect to their cores is kept at very low temperature by a double thermostat. ${ }^{56}$ In this manner, the trajectory of the Drude particles follows closely that of the relaxed Drude dipoles. In this work, the degrees of freedom of DPs with respect to their DC were kept at $1 \mathrm{~K} .{ }^{46,56}$

It was recently shown ${ }^{50}$ that such dual-thermostat method may not ensure perfect equipartition between the all molecular DOF, and a new thermostat handling the molecular center-of-mass motion and the intramolecular DOF separately was proposed. We implemented this temperature-grouped Nosé-Hoover thermostat in the LAMMPS $^{45}$ molecular dynamics package (as part of the USER-DRUDE package).

\section{Force field specification}

Bonds terminating in hydrogen atoms were constrained using the SHAKE algorithm. We represent cholinium, ammonium, $\mathrm{Cl}^{-}$, bis(fluorosulfonyl)imide (FSI) and bis(trifluoromethansulfonyl)imide $\left(\mathrm{Ntf}_{2}^{-}\right.$or TFSI) with the $\mathrm{CL} \& \mathrm{Pol}^{25}$ force field for ionic liquids. For $\mathrm{Li}^{+}$, $\mathrm{NO}_{3}^{-}$and ethylene glycol, we derived a polarizable version of OPLS-AA, ${ }^{44}$ following a route analogous to the one of CL\&Pol. Atomic partial charges in nitrate and ethylene glycol were computed, on geometries optimised with Gaussian, ${ }^{57}$ using the CHelpG ${ }^{58}$ method with MP2/ccpVTZ(-f) electron densities.

Atomic polarizabilities were taken from recent publications. ${ }^{23,59,60}$ Only heavy atoms were treated as polarizable, with the polarizabilities of $\mathrm{H}$ atoms summed onto the atoms to which they are bound. The mass of the $\mathrm{DP}$ was set to $m_{D}=0.4 \mathrm{u}$ and the force constant of the DC-DP harmonic bond was fixed at $k_{D}=4184 \mathrm{~kJ} \mathrm{~mol}^{-1}$. The partial charges of DPs were derived from atomic polarizabilities according to $\alpha=q_{D}^{2} / k_{D} \cdot{ }^{56}$ The short-range 
dipole-dipole electrostatic interactions were reduced using a Thole damping function ${ }^{32,46}$ with parameter $a=$ $2.6 . .^{19}$

The non-bonded attractive energies of the original, fixed-charge force fields were modified to avoid double counting of polarization effects. ${ }^{26}$ The scaling factors were evaluated mostly using the fragment-based predictive scheme proposed in our previous work, ${ }^{25}$

$$
k_{i j}=\left(1+c_{0} r_{i j}^{2} \frac{Q_{i}^{2} \alpha_{j}+Q_{j}^{2} \alpha_{i}}{\alpha_{i} \alpha_{j}}+c_{1} \frac{\mu_{i}^{2} \alpha_{j}+\mu_{j}^{2} \alpha_{i}}{\alpha_{i} \alpha_{j}}\right)^{-1}
$$

where the $\alpha$ are the polarizabilities of the fragments, $Q$ their net charges, $\mu$ their dipole moments, $r_{i j}$ is the equilibrium distance of a given fragment dimer, and $c_{0}=0.25$ and $c_{1}=0.11$ are fitted coefficients. This predictive scheme substitutes for case-by-case SAPT calculations, which are costly. Nonetheless, the scaling factor for EAN system was derived here at the SAPT $2+/$ aDZ level $^{25}$ because we consider that this is a archetypal protic ionic liquid, formed by small ions and the $\mathrm{NO}_{3}^{-}$fragment was not yet described in CL\&Pol. We thus opted for a firstprinciples calculation of the respective scaling factors.

According to the fragment approach, ethylene glycol (EG) was split into two methanol (MeOH) units, triethylammonium $\left(\mathrm{N}_{2220}^{+}\right)$was represented by trimethylammonium $\left(\mathrm{N}_{1110}^{+}\right)$and butane $\left(\mathrm{C}_{4} \mathrm{H}_{10}\right)$, whereas cholinium $\left(\mathrm{Ch}^{+}\right)$, ethylammonium $\left(\mathrm{N}_{2000}^{+}\right)$, 1-ethyl3-methylimidazolium $\left(\mathrm{C}_{2} \mathrm{C}_{1} \mathrm{im}^{+}\right), \mathrm{Ntf}_{2}^{-}, \quad \mathrm{FSI}^{-}$and $\mathrm{NO}_{3}^{-}$were treated as single fragments. The scaling coefficients for Lennard-Jones $\epsilon$ parameters are reported in Table I; the corresponding $\sigma$ parameters were kept unchanged (if not mentioned below). The Lennard-Jones parameters of $\mathrm{Li}^{+}$were not modified because of the small polarizability of the cation, $\alpha_{\mathrm{Li}^{+}}=0.03 \AA^{-3}$.

TABLE I. Scaling coefficients for the modification of nonbonded attractive interactions

\begin{tabular}{llll}
\hline System & Fragment 1 & Fragment 2 & $k$ \\
\hline Ethylene glycol & $\mathrm{MeOH}$ & $\mathrm{MeOH}$ & 0.87 \\
ChCl-EG & $\mathrm{Ch}^{+}$ & $\mathrm{Cl}^{-}$ & 0.48 \\
& $\mathrm{Ch}^{+}$ & $\mathrm{MeOH}$ & 0.66 \\
& $\mathrm{Cl}^{-}$ & $\mathrm{MeOH}$ & 0.60 \\
& $\mathrm{MeOH}$ & $\mathrm{MeOH}$ & 0.87 \\
EAN & $\mathrm{N}_{2000}^{+}$ & $\mathrm{NO}_{3}^{-}$ & 0.20 \\
{$\left[\mathrm{~N}_{2220}\right]\left[\mathrm{NTf}_{2}\right]$} & $\mathrm{N}_{1110}^{+}$ & $\mathrm{C}_{4} \mathrm{H}_{10}$ & 0.60 \\
& $\mathrm{~N}_{1110}^{+}$ & $\mathrm{NTf}_{2}^{-}$ & 0.50 \\
& $\mathrm{C}_{4} \mathrm{H}_{10}$ & $\mathrm{NTf}_{2}^{-}$ & 0.69 \\
& $\mathrm{C}_{4} \mathrm{H}_{10}$ & $\mathrm{C}_{4} \mathrm{H}_{10}$ & 1.00 \\
Li[FSI]-[C $\mathrm{C}_{2}$ im] $]$ FSI] & $\mathrm{C}_{2} \mathrm{C}_{1} \mathrm{im}^{+}$ & $\mathrm{FSI}^{-}$ & 0.66 \\
\hline
\end{tabular}

\section{POLARIZABLE FORCE FIELD DEVELOPMENT}

\section{A. Charge-dipole damping function}

Interactions between induced dipoles are dampened at short range through Thole functions, thus avoiding instabilities in molecular systems or in aprotic ionic liquids. However, in the systems studied in this work, the presence of strong interactions between induced dipoles and certain charges, namely "naked" $\mathrm{H}$ atoms involved in $\mathrm{H}$ bonds or very small ions, posed additional issues to the stability of trajectories, as discussed above in the introduction. The occurrence of these instabilities prompted us to also apply short-range damping (or smearing) to charge-induced dipole interactions. Our choice of shortrange damping functions was guided by the literature on polarizable simulations of molten salts, ${ }^{39-43}$ where small monoatomic ions are common. The functional form of the Tang-Toennies damping functions, ${ }^{38}$ originally devised for dispersion interactions, is kept,

$$
f_{n}(r)=1-c \cdot e^{-b r} \sum_{k=0}^{n} \frac{(b r)^{k}}{k !}
$$

where $b$ is a parameter determining the range below which the interactions are damped (Figure 1), but for charge-dipole interactions the sum goes to order $n=4$. We chose to keep $c=1$ so that $f_{n}(r) \rightarrow 0$ when $r \rightarrow 0$.

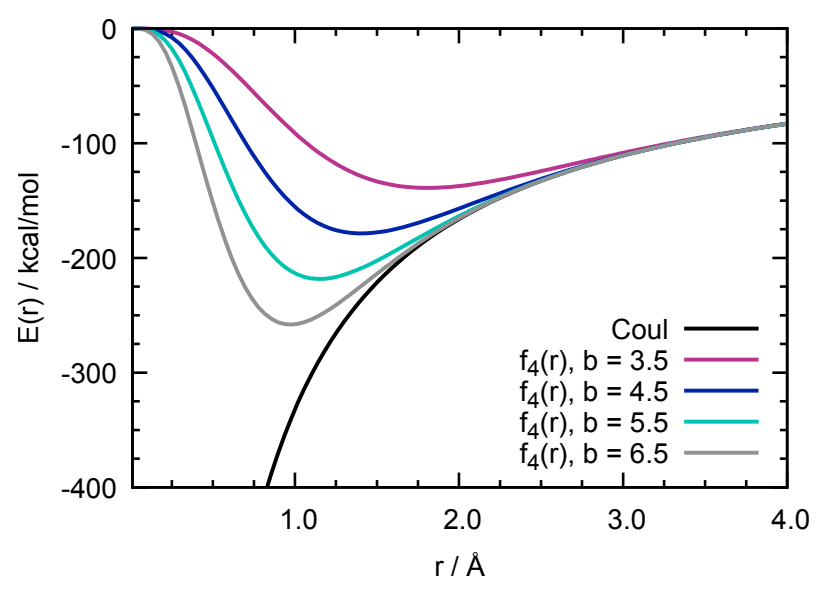

FIG. 1. Electrostatic potential energy of two opposite unit charges without damping (black) and with damping by the Tang-Toennies function with different ranges determined by the $b$ parameter.

The Tang-Toennies damping function requires adjustment of the $b$ parameter that sets the range of the damping/smearing effect. In the original studies on molten salts, the $b$ parameter was obtained through forcematching based on first-principle calculations. ${ }^{41} \mathrm{~A}$ detailed parameterisation for the more complex molecular ions of the present systems would imply very high computational costs. Therefore, we consider the $b$ parameter as universal and empirical, and make use of 
the Tang-Toennies damping function as a safeguard preventing a "polarization catastrophe", rather than as an accurate representation of very short-range electrostatic forces. In strongly H-bonded systems, the Tang-Toennies function (for charge-dipole short-range damping) should be used in conjunction with Thole function (for dipoledipole damping in general polarizable simulations).

Ethylene glycol, the simplest compound forming both intra- and intermolecular hydrogen bonds and a component of common DES, and which is challenging for polarizable simulations, was chosen as a test for a scan of the $b$ parameter in the range $3.5-6.5$. The damping function was applied to the interactions between the $\mathrm{H}$ atoms of the $\mathrm{OH}$ and all induced dipoles present in the system. Too small $b$ values lead to the excessive weakening of the intermolecular forces between the nearest neighbours, bringing a unbalance to the non-bonded interactions and affecting the properties of the system as presented in Table II. Too big $b$ values cause a $0.2-0.3 \AA$ displacement of the oxygen's DPs from its DCs, towards the hydrogen atoms, and such strong dipoles freeze the system and increase its density. The value $b=4.5$ was found to be optimum, reproducing well both equilibrium and dynamic properties of ethylene glycol.

TABLE II. Experimental ${ }^{61,62}$ and calculated properties of ethylene glycol at $298 \mathrm{~K}$ at different values of the $b$ parameter in the Tang-Toennies damping function.

\begin{tabular}{|c|c|c|c|c|c|c|}
\hline & $b$ & $\rho$ & $\rho^{\mathrm{dev}}$ & $D$ & $\eta^{\mathrm{eq}}$ & $\eta^{\text {noneq }}$ \\
\hline Exp & & 1.110 & & 0.9 & 16.63 & \\
\hline FixQ & & 1.071 & -3.5 & 2.8 & $5 \pm 1$ & \\
\hline \multirow[t]{7}{*}{ Drude NH } & 3.5 & 1.142 & 2.6 & 1.6 & $10 \pm 2$ & \\
\hline & 4.0 & 1.117 & 0.6 & 2.3 & $6 \pm 1$ & \\
\hline & 4.5 & 1.116 & 0.5 & 1.6 & $10 \pm 2$ & \\
\hline & 5.0 & 1.124 & 1.3 & 0.53 & $38 \pm 10$ & \\
\hline & 5.5 & 1.132 & 2.0 & 0.11 & $440 \pm 237$ & \\
\hline & 6.0 & 1.132 & 2.0 & 0.03 & $1791 \pm 381$ & \\
\hline & 6.5 & 1.132 & 2.0 & 0.02 & $2977 \pm 1803$ & \\
\hline Drude $\operatorname{tgNH}$ & 4.5 & 1.116 & 0.6 & 1.3 & $11 \pm 3$ & $8.6 \pm 0.3$ \\
\hline
\end{tabular}

\section{B. Modification of atomic diameters}

The absence of Lennard-Jones sites on certain hydrogen atoms can lead in the worse cases to instabilities of the simulations, which we could mitigate through chargedipole damping, but can also result in significant structural changes upon introduction of polarization. Doubly ionic $\mathrm{H}$-bonds, ${ }^{63}$ formed between a cation and an anion, could undergo significant strengthening, "freezing" the system, which we observed in EAN. The H-bond formed between oxygen atoms of nitrate and hydrogen atoms of the ammonium head group, $\mathrm{O}_{\mathrm{N}}-\mathrm{H}_{\mathrm{N}}$, is shortened to
$1.2 \AA$ with an increased intensity of the first RDF peak up to 14.5 (Figure 2). The peak position is comparable with the intramolecular $\mathrm{N}_{1}-\mathrm{H}_{\mathrm{N}}$ distance and differs significantly from the 1.8-1.9 $\AA$ obtained from ab-initio MD. ${ }^{9-11}$ Since the hydrogen atoms are just point charges embedded into their neighbouring nitrogen atoms, the repulsive potential between $\mathrm{N}_{1}$ and $\mathrm{O}_{\mathrm{N}}$ are not sufficient to compensate the increased polarization effects. Enhancement of repulsion forces between cation and anion was achieved through modification of $\sigma_{\mathrm{ON}-\mathrm{N} 1}$, which shifts the 1 st RDF peak to $1.9 \AA$ and decreases its intensity, as illustrated in Figure 2.

In order to choose the optimal $\sigma_{\mathrm{ON}-\mathrm{N} 1}$ value, we evaluated the ability of the models with $\sigma$ in the range 3.1$4.0 \AA$ to predict properties of EAN correctly as presented in Table III. The densities computed with the models with $\sigma$-ranges of $3.20-3.25 \AA$ and $3.75-3.80 \AA$ were the closest to experiment, but the observed local structures were completely different, this having a strong impact on dynamics. Thus, the final value of $\sigma_{\mathrm{ON}-\mathrm{N} 1}=3.75 \AA$ was chosen because it improves structure and dynamics, and gives transport properties in reasonable agreement with experiment, preventing "freezing" of the system.

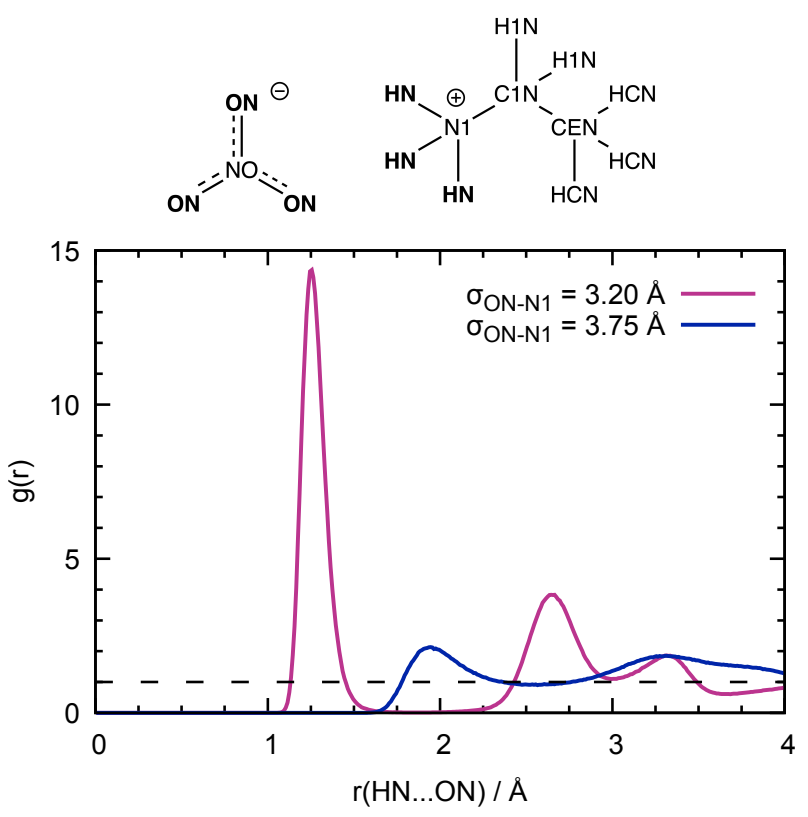

FIG. 2. Radial distribution function of $\mathrm{O}_{\mathrm{N}}-\mathrm{H}_{\mathrm{N}}$ in EAN with different $\sigma$ Lennard-Jonnes interaction parameters. The rose curve corresponds to an unchanged EAN force field with polarization terms added; the blue curve corresponds to modified Lennard-Jones $\sigma_{\mathrm{ON}-\mathrm{N} 1}$ parameter.

The same issue was observed in choline chrolide plus ethylene glycol ( $\mathrm{ChCl}-\mathrm{EG})$, as presented in Figure 3, where the peaks of the RDFs between $\mathrm{Cl}^{-}$and the $\mathrm{H}_{\mathrm{O}}$ atoms of cholinium or ethylene glycol were shifted to a very short distance of $1.7 \AA$ and reached the intensity of $38 \mathrm{a} \mathrm{u}$ and $18 \mathrm{au}$, respectively. As in the previous case, an increase of $\sigma_{\mathrm{O}-\mathrm{Cl}}$ from $3.37 \AA$ to $3.70 \AA$ allowed us 
TABLE III. Experimental ${ }^{7,64}$ and calculated equilibrium and dynamic properties of EAN at $298 \mathrm{~K}$ at different $\sigma_{O N-N 1}$ values.

\begin{tabular}{|c|c|c|c|c|c|c|}
\hline & $\sigma_{O N-N 1}$ & $\rho$ & $\rho^{\mathrm{dev}}$ & $D_{\text {cat }}$ & $D_{\text {ani }}$ & $\eta^{\mathrm{eq}}$ \\
\hline Exp & & 1.210 & & 3.86 & 7.80 & 32.7 \\
\hline \multirow[t]{10}{*}{ Drude NH } & 3.10 & 1.181 & -2.36 & & & \\
\hline & 3.15 & 1.184 & -2.15 & & & \\
\hline & 3.20 & 1.206 & -0.28 & 0.05 & 0.08 & $2964 \pm 3602$ \\
\hline & 3.25 & 1.213 & 0.32 & & & \\
\hline & 3.35 & 1.261 & 4.24 & & & \\
\hline & 3.50 & 1.267 & 4.77 & & & \\
\hline & 3.70 & 1.228 & 1.54 & & & \\
\hline & 3.75 & 1.217 & 0.61 & 4.15 & 6.92 & $52 \pm 35$ \\
\hline & 3.80 & 1.205 & -0.41 & 5.22 & 6.88 & $64 \pm 19$ \\
\hline & 4.00 & 1.147 & -5.21 & & & \\
\hline
\end{tabular}

Units are: $\sigma / \AA, \rho / \mathrm{g} \mathrm{cm}^{-3}, \rho^{\mathrm{dev}} / \%, D / 10^{-11} \mathrm{~m}^{2} \mathrm{~s}^{-1}$, $\eta / \mathrm{mPas}$. The Yeh-Hummer correction to diffusion coefficients was not applied at this stage.

to obtain a reasonable structure, details of which will be presented in the following section.

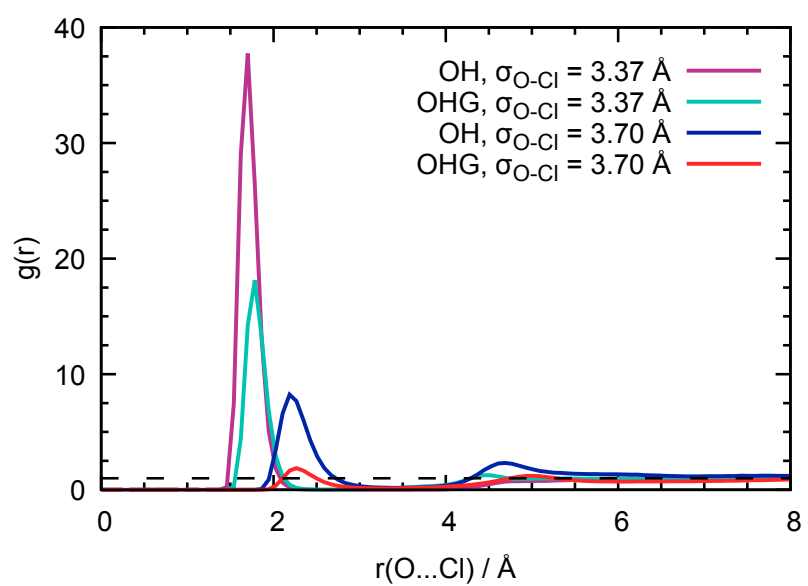

FIG. 3. Radial distribution function of $\mathrm{O}-\mathrm{Cl}$ in $\mathrm{ClCl}+\mathrm{EG}$ with different values of the Lennard-Jonnes $\sigma$ parameter. $\mathrm{H}_{\mathrm{O}}$ is the hydrogen atom of a hydroxyl group of choline; $\mathrm{H}_{\mathrm{OG}}$ in $\mathrm{OH}$ groups of ethylene glycol. The $\sigma_{\mathrm{O}-\mathrm{Cl}}$ value of $3.37 \AA$ corresponds to the unchanged EAN force field while $3.70 \AA$ is the new value.

Contrary to the above two cases, no adjustments are required for the protic ionic liquid triethylammonium bis(trifluoromethanesulfonyl)amide, $\quad\left(\left[\mathrm{N}_{2220}\right]\left[\mathrm{NTf}_{2}\right]\right)$, where the ammonium hydrogen atom is shielded by the side chains, making more difficult the close approach of the voluminous anion.

\section{Prediction of properties of PIL, DES and electrolytes}

A variety of systems were chosen to validate the new polarizable force field: protic ionic liquids, ethylammonium nitrate (EAN) and triethylammonium bis(trifluoromethanesulfonyl)amide $\left(\left[\mathrm{N}_{2220}\right]\left[\mathrm{NTf}_{2}\right]\right)$; a deep eutectic solvent, choline chrolide + ethylene glycol (ChCl-EG, 1:2); and a lithium-based electrolyte consisting of lithium bis(fluorosulfonyl)imide (Li[FSI]) and 1-ethyl-3-methylimidazolium bis(fluorosulfonyl)imide $\left(\left[\mathrm{C}_{2} \mathrm{C}_{1} \mathrm{im}\right][\mathrm{FSI}]\right)$. The structural formulae of the selected compounds are given in Figure 4. The calculation of equilibrium and dynamic properties were carried out at different temperatures (EAN at $260 \mathrm{~K}$ and $298 \mathrm{~K},\left[\mathrm{~N}_{2220}\right]\left[\mathrm{NTf}_{2}\right]$ at $298 \mathrm{~K}$ and $\left.340 \mathrm{~K}\right)$ or at different compositions ( $\mathrm{Li}[\mathrm{FSI}]-\left[\mathrm{C}_{2} \mathrm{C}_{1} \mathrm{im}\right][\mathrm{FSI}]$ at mole fractions of lithium salt of 0.2 and 0.4 ), according to available experimental data.

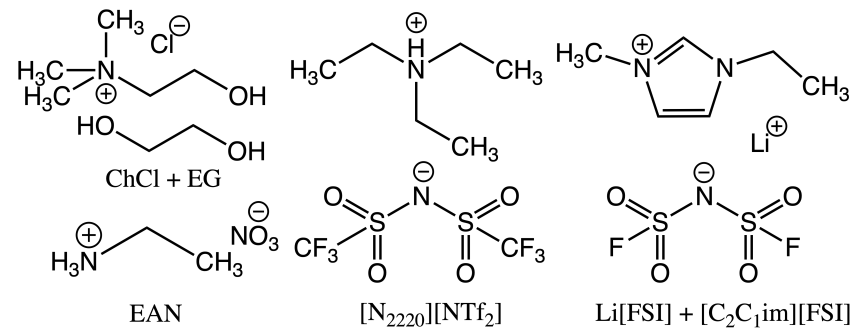

FIG. 4. The structural formulae of the selected compounds used in the validation of the force field.

Density and dynamic properties of EAN predicted by polarizable simulations using both the traditional $(\mathrm{NH})$ and the temperature-grouped (tgNH) Nosé-Hoover thermostats are in good agreement with experimental data, as reported in Table IV. Non-equilibrium MD simulations with the periodic perturbation method coupled with the $\operatorname{tg} \mathrm{NH}$ thermostat improve the prediction of viscosity of EAN. The CL\&Pol force field describes the properties of $\mathrm{ChCl}-\mathrm{EG}$ well, although the diffusion coefficient of the H-bond donor (EG) is overestimated. The same is observed for pure ethylene glycol (Table II), with an increase the polarizable version of OPLS-AA force field. Transport properties of $\left[\mathrm{N}_{2220}\right]\left[\mathrm{NTf}_{2}\right]$ are relatively well reproduced, while the density values are decreased by $2 \%$. This effect of the density deviation upon introducing polarization in pure ionic liquids was discussed in our previous paper ${ }^{25}$ and can be fixed by reducing the diameter of LJ sites $\sigma$ by $1.5 \%$.

The Cl\&Pol force field is able to maintain the crystal structure of a solid EAN, as presented in the Table V. Most unit-cell parameters are in good agreement with experiment, except $b$ and $\beta$, the drift of which leads to an underestimation of the density, which we considered as acceptable for a force field mainly aimed at liquid systems.

A correct description of the micro-structure of strong $\mathrm{H}$-bonded systems is a criterion of reliability with respect to the use of the Tang-Toennies damping function in the CL\&Pol force field. Medium-strength hydrogen bonds are formed between the hydroxyl groups in ethylene glycol, and also between the acidic hydrogen of the cation and the oxygen atom of the anion in 
TABLE IV. Experimental ${ }^{7,64-67}$ and calculated equilibrium and dynamic properties of PIL and DES.

\begin{tabular}{|c|c|c|c|c|c|c|c|}
\hline & $\rho$ & $\rho^{\operatorname{dev}}$ & $D_{\text {cat }}$ & $D_{\text {ani }}$ & $D_{\mathrm{HBD}}$ & $\eta^{\mathrm{eq}}$ & $\eta^{\text {noneq }}$ \\
\hline \multicolumn{8}{|c|}{ EAN $298 \mathrm{~K}$} \\
\hline Exp & 1.210 & & 3.86 & 7.80 & & 32.7 & \\
\hline Drude NH & 1.217 & 0.6 & 4.57 & 7.35 & & $52 \pm 35$ & \\
\hline Drude tgNH & 1.219 & 0.8 & 4.04 & 5.95 & & $64 \pm 20$ & $32.8 \pm 1.5$ \\
\hline \multicolumn{8}{|c|}{$\mathrm{ChCl}-\mathrm{EG} 298 \mathrm{~K}$} \\
\hline Exp & 1.120 & & 2.62 & & 4.77 & 37 & \\
\hline Drude NH & 1.129 & 0.8 & 1.92 & 5.29 & 14.1 & $73 \pm 36$ & \\
\hline \multirow{2}{*}{\multicolumn{8}{|c|}{$\left[\mathrm{N}_{2220}\right]\left[\mathrm{Ntf}_{2}\right] 298 \mathrm{~K}$}} \\
\hline & & & & & & & \\
\hline Drude NH & 1.390 & -2.1 & 2.03 & 2.24 & & $56 \pm 15$ & \\
\hline Drude $\operatorname{tgNH}$ & 1.392 & $\begin{array}{r}-1.9 \\
{\left[\mathrm{~N}_{2}\right.}\end{array}$ & $\begin{array}{c}2.34 \\
220][\mathrm{N}\end{array}$ & $\begin{array}{r}2.34 \\
\left.\mathrm{Ttf}_{2}\right] 3\end{array}$ & $40 \mathrm{~K}$ & $48 \pm 7$ & $32.3 \pm 1.3$ \\
\hline Exp & & & 12.7 & 9.7 & & & \\
\hline Drude NH & 1.343 & & 9.70 & 8.64 & & $33 \pm 21$ & \\
\hline Drude tgNH & 1.345 & & 9.35 & 8.40 & & $20 \pm 5$ & $9.8 \pm 0.2$ \\
\hline
\end{tabular}

TABLE V. The experimental ${ }^{68}$ and calculated properties of ethylammonium nitrate crystal at $260 \mathrm{~K}$.

\begin{tabular}{lcccccccc}
\hline & $\rho$ & $\rho^{\text {err }}$ & $a$ & $b$ & $c$ & $\alpha$ & $\beta$ & $\gamma$ \\
\hline Exp & 1.367 & & 39.6 & 46.1 & 39.9 & 90.0 & 112.7 & 90.0 \\
Drude NH & 1.255 & -8.2 & 39.5 & 50.0 & 39.4 & 90.0 & 110.3 & 90.1 \\
Drude tgNH & 1.253 & -8.3 & 39.6 & 50.0 & 39.4 & 90.0 & 110.6 & 90.0 \\
\hline \multicolumn{4}{c}{ Units are: } & $\rho / \mathrm{g} \mathrm{cm}^{-3}, \rho^{\mathrm{dev}} / \%,(a, b, c) / \AA$. & &
\end{tabular}

$\left[\mathrm{N}_{2220}\right]\left[\mathrm{NTf}_{2}\right]$, as illustrated in Figure 6 . The $\mathrm{H} \cdots \mathrm{A}$ distances and the $\mathrm{D}-\mathrm{H} \cdots \mathrm{A}$ angles (where $\mathrm{A}$ is a H-bond acceptor atom and $\mathrm{D}$ the atom attached to the donor hydrogen) are in agreement with theoretical studies of similar systems. ${ }^{63}$ The presence of the stronger H-bonds in the PIL when compared to EG can be explained by the enhanced cation-anion electrostatic attraction.

The Cl\&Pol force field predicts a linear hydrogen bond with an average distance of $1.9 \AA$ and an angle of $160^{\circ}$ in EAN (Figures 2 and 6). This agrees with $a b$-initio MD studies of alkylammonium nitrates with the Car-Parrinello method ${ }^{10,11}$ and also with density functional-based tight-binding methods. ${ }^{9}$ The linearity of the hydrogen bond was confirmed experimentally by X-ray diffraction on the crystal. ${ }^{68}$ By means of the empirical potential structure refinement (EPSR) applied to X-ray and neutron scattering data on liquid EAN, several groups ${ }^{4-7}$ proposed the formation of a bent H-bond, with a length of $2.4 \AA$ and an angle of $109^{\circ}$, where three hydrogen atoms of the ammonium head group share one oxygen atom of the anion. This is a result of the presence of a Lennard-Jones site of diameter $2.5 \AA$ on each hydrogen atom in the force field used for the EPSR. Repeating the same procedure with a model where hydrogen atoms are embedded into the neighbouring nitrogen atom $\left(\sigma_{\mathrm{H}}=0.8 \AA\right)$, followed by MD simulation with a three- body term included, led to a linear, directional hydrogen bond with a distance of $1.9-2.0 \AA .^{8}$ The ambiguous interpretation of X-ray and neutron scattering data of the liquid system compromises the reliability of the potential fitting procedure. We consider the that the linear description of the hydrogen bond in EAN has stronger physical background and our model, being compatible with a directional H-bond, predicts equilibrium and dynamic properties of EAN reasonably well. Therefore, the present polarizable simulations contribute to the analysis of H-bond patterns in EAN, one of the most important protic ionic liquids, both in fundamental and applied research.

Next, the liquid structure of EAN was studied by computing static structure factors that are compared to Xray and neutron scattering data in Figure 5. Both Xray and neutron structure factors are in good agreement with experimental results, with divergences observed for $q$ values smaller than $1 \AA^{-1}$ for hydronen-rich samples, which could be explained by residual inelastic scattering of light $\mathrm{H}$ atoms in the experimental data, not fully removed by correction methods. ${ }^{4}$ The main characteristic peak at $1.71 \AA^{-1}$ with a Bragg spacing of $3.7 \AA$ corresponds to cation-anion separations ${ }^{8}$ is in accordance with the experimental peaks near $1.6 \AA^{-1} .6,69,70$ In the d3-EAN sample, simulations produce a clear sharp peak at $0.64 \AA^{-1}$, corresponding to a Bragg spacing of $9.8 \AA$, evidence of long range ordering in the liquid. This coincides with experimental neutron scattering peaks at $0.625 \AA^{-14}$ and $0.66 \AA^{-1} .{ }^{71}$ This feature also appears as a small pre-peak in X-ray scattering at $0.6 \AA^{-1}, 6,69,70$ whereas in our simulation it is split into three separate contributions at $0.42 \AA^{-1}, 0.69 \AA^{-1}$ and $1.0 \AA^{-1}$.

The ChCl-EG DES differs from the previous systems in which there is no competition for the H-bonding sites. In this DES, the dominant interactions are those of $\mathrm{Cl}^{-}$ with $\mathrm{H}$ atoms of cholinium and of ethylene glycol (Figure 6). The strength of these interactions decreases in the order $\mathrm{H}_{\mathrm{O}}\left(\mathrm{Ch}^{+}\right)>\mathrm{H}_{\mathrm{O}}(\mathrm{EG}) \gg \mathrm{H}_{\mathrm{C}}\left(\mathrm{Ch}^{+}\right)$. Also, and due to the high concentration of EG (2:1 mole ration with respect to $\mathrm{ChCl}$ ), intermolecular interactions between ethylene glycol molecules are important. The interactions between the cation and the HBD are not marked, as shown in Figure 7. These results are in agreement with a recent ab-initio MD study, ${ }^{72}$ even though the intensities of certain peaks do not coincide perfectly, as can be expected due to the small sizes and duration sof AIMD trajectories, as well as the obvious differences in the Hamiltonian. The positions of the first peaks in the $\mathrm{H}_{\mathrm{O}}-\mathrm{Cl}, \mathrm{H}_{\mathrm{OG}}-\mathrm{Cl}$ and $\mathrm{H}_{1}-\mathrm{Cl}$ RDFs are $2.19 \AA, 2.27 \AA$ and $2.67 \AA$ from the CL\&Pol force field, and $2.10 \AA, 2.14 \AA$ and $2.75 \AA$ from AIMD, which is quite close. The peak intensities are in good accord between two methods for $\mathrm{Cl}^{-} \ldots \mathrm{Ch}^{+}$correlations, while the intensity for EG is lower from our force field when compared to AIMD, which could be related to faster diffusion of the HBD discussed above. 


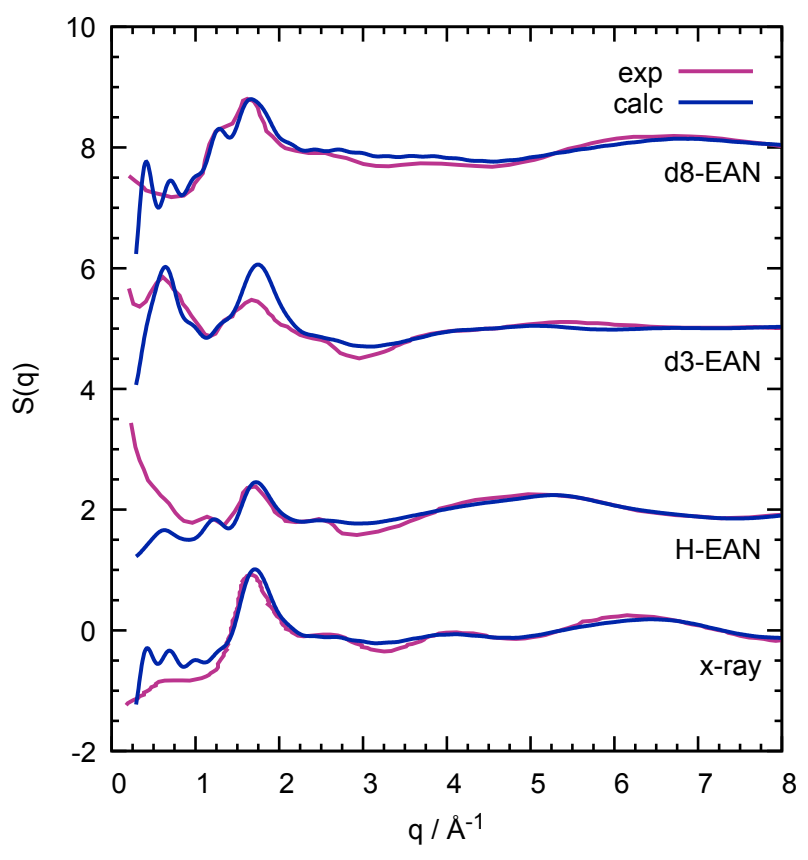

FIG. 5. Experimental ${ }^{4,6}$ and calculated structure factor of liquid EAN at $298 \mathrm{~K}$; from bottom to top: x-ray diffraction, neutron diffraction data of H-EAN, d3-EAN (deuterated ammonium group), d8-EAN (fully deuterated cation) with the respective vertical offset of $0.0,2.0,5.0$ and 8.0.

A lithium-based electrolyte was the next system of interest to validate the CL\&Pol force field with the TangToennies damping function. In the mixture of the ionic liquid with the salt, $\mathrm{Li}[\mathrm{FSI}]-\left[\mathrm{C}_{2} \mathrm{C}_{1} \mathrm{im}\right][\mathrm{FSI}]$, the density values (Table VI) are reproduced within $1 \%$ for both compositions when using the traditional NH thermostat, while the $\operatorname{tgNH}$ thermostat leads to slightly higher densities, by $1.5 \%$, which is quite acceptable. Viscosity values are overestimated, especially at higher lithium salt content, which can be due to the OPLS-AA model for lithium and to the approximations used during the fit of the Green-Kubo relation. The classical NH thermostat shows better performance than the tgNH one due to its tendency to fluidize the system (caused by incorrect kinetic energy distribution) that compensates artefacts of the force field. The lack of experimental data on diffusion coefficients does not allow us to estimate the accuracy of the transport properties, but the general trends are in agreement with earlier work. ${ }^{73}$

The strong interaction between $\mathrm{Li}^{+}$and the $\mathrm{O}$ atom of the anion is confirmed by the radial distribution functions (Figure 8). The main peak of the RDF is at $2.1 \AA$ with an intensity of 22.7 , and the corresponding coordination number is $4.5 \mathrm{O}$ atoms in the first solvation shell around $\mathrm{Li}^{+}$. This interaction dominates over the interaction of the anion with the $\mathrm{H}$ atoms of the imidazolium cation, where the intensity of the first peak does not exceed 2. A similar behavior of $\mathrm{Li}^{+}$is observed in the
TABLE VI. Experimental ${ }^{74}$ and calculated equilibrium and dynamic properties of $[\mathrm{Li}][\mathrm{FSI}]-\left[\mathrm{C}_{2} \mathrm{C}_{1} \mathrm{im}\right][\mathrm{FSI}]$ at $338 \mathrm{~K}$.

\begin{tabular}{|c|c|c|c|c|c|c|c|}
\hline & $\rho$ & $\rho^{\mathrm{dev}}$ & $D_{\mathrm{Li}^{+}}$ & $D_{\mathrm{im}^{+}}$ & $D_{\text {ani }}$ & $\eta^{\mathrm{eq}}$ & $\eta^{\text {non-eq }}$ \\
\hline \multicolumn{8}{|c|}{$x_{[\mathrm{Li}][\mathrm{FSI}]}=0.2$} \\
\hline Exp & 1.460 & & & & & 11.1 & \\
\hline Drude NH & 1.461 & 0.03 & 4.48 & 8.36 & 6.24 & $21 \pm 3$ & \\
\hline Drude $\operatorname{tg} \mathrm{NH}$ & 1.480 & 1.34 & 2.73 & 4.15 & 3.47 & $40 \pm 3$ & $33.3 \pm 1.3$ \\
\hline \multicolumn{8}{|c|}{$x_{[\mathrm{Li}][\mathrm{FSI}]}=0.4$} \\
\hline Exp & 1.536 & & & & & 21.8 & \\
\hline Drude NH & 1.546 & 0.66 & 1.69 & 3.11 & 1.83 & $118 \pm 61$ & \\
\hline Drude $\operatorname{tgNH}$ & 1.563 & 1.76 & 0.74 & 1.18 & 0.77 & $676 \pm 417$ & $143 \pm 10$ \\
\hline
\end{tabular}

Units are: $\rho / \mathrm{g} \mathrm{cm}^{-3}, \rho^{\mathrm{dev}} / \%, D / 10^{-11} \mathrm{~m}^{2} \mathrm{~s}^{-1}, \eta / \mathrm{mPas}$.

$\mathrm{Li}\left[\mathrm{Ntf}_{2}\right]-\left[\mathrm{C}_{6} \mathrm{C}_{1} \mathrm{im}\right]\left[\mathrm{Ntf}_{2}\right]$ system with a peak of intensity 20 at $1.9 \AA .73$

\section{CONCLUSIONS}

We extended the transferable, polarizable CL\&Pol force field, initially developed for general aprotic ionic liquids, to systems containing strong hydrogen bonds or small ions with high charge density. These systems cause trajectory stability issues in polarizable simulations due to the strong pull of the small ions or of ionic H-bonds on the Drude induced dipoles. We found that a damping, or smearing, function acting at short range between those problematic charged sites and the Drude induced dipoles was neeeded in order to stabilize the trajectories, preventing the "polarization catastroph".

Inspired by work in the literature on molten salts, we applied a Tang-Toennies damping function to the interaction of Drude dipoles in those specific cases. The parameters of the damping function were validated through analyses of the structure and dynamics of five fundamentally different systems: protic ionic liqudis, deep eutectic solvents, and a lithium in ionic liquid electrolyte. Comparison with experiment showed good prediction of density, viscosity, ion diffusion coefficients and correct description of the microscopic structure.

We also compared the performance of traditional and temperature-grouped Nosé-Hoover thermostats for modelling ionic and ion-molecular systems, and the use of the latter is strongly recommended due to the correct treatment of translational, intramolecular and polarization degrees of freedom. At present, this thermostat is available in the OpenMM and LAMMPS molecular dynamics codes.

We recommend the periodic perturbation method of non-equilibrium MD to calculate viscosity of ionic fluids, since it gives better predictions with a tighter confidence interval than using the Green-Kubo relation: fitting the tail of the auto-correlation function leads to large uncertainties even if long trajectories are used, and fitting of the "running integral" requires averaging over hundreds of independent equilibrium trajectories. 


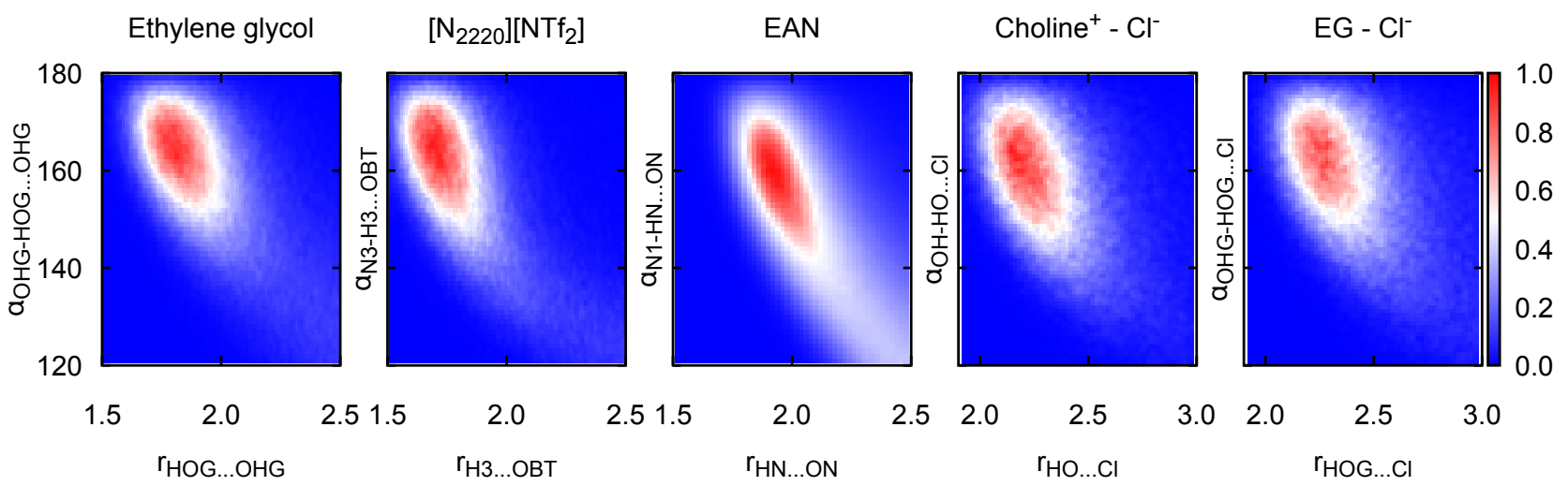

FIG. 6. Probability contours revealing hydrogen bonds in ethylene glycol, $\left[\mathrm{N}_{2220}\right]\left[\mathrm{NTf}_{2}\right]$, EAN and ChCl-EG. The $x$-axes represent the distances between the $\mathrm{H}$ atoms and the acceptor atoms. The $y$-axes represent the angles formed by the $\mathrm{D}-\mathrm{H} \cdot \cdots \mathrm{A}$ hydrogen bonds, where $\mathrm{D}$ is a donor atom attached to the hydrogen. The $\mathrm{N}_{3}$ and $\mathrm{H}_{3}$ atomic labels in the second plot correspond to $\mathrm{H}$ boud to $\mathrm{N}$ of the ammonium head group, and $\mathrm{O}_{\mathrm{BT}}$ are $\mathrm{O}$ atoms of $\mathrm{NTf}_{2}^{-}$. The remaining labels are explained in the molecular structures within this text.
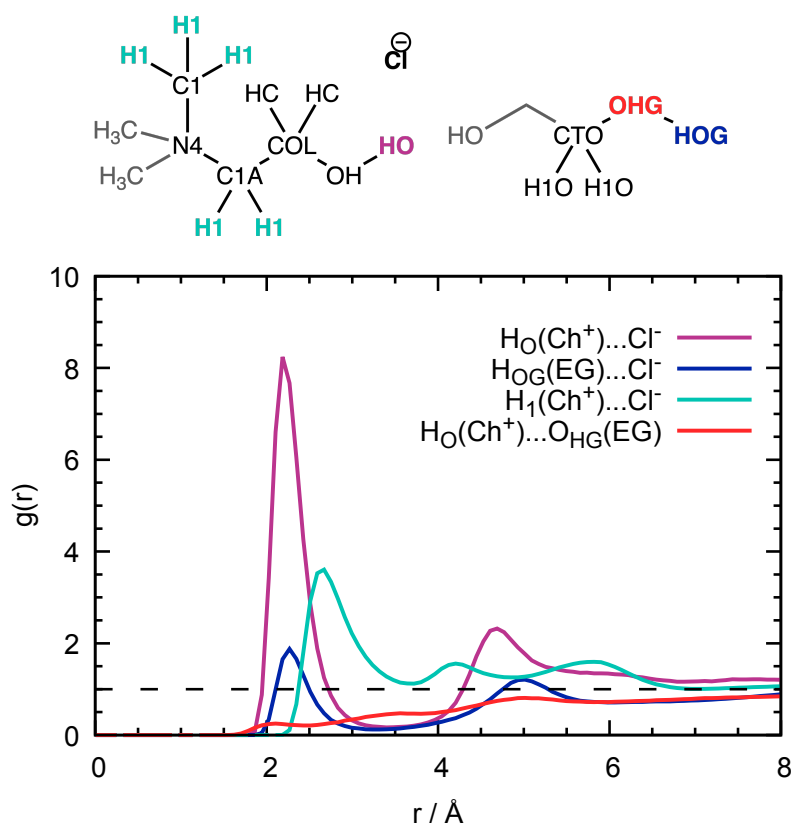

FIG. 7. Radial distribution functions of $\mathrm{Cl}-\mathrm{HO}, \mathrm{Cl}-\mathrm{H}_{\mathrm{OG}}$, $\mathrm{Cl}-\mathrm{H}_{1}, \mathrm{O}_{\mathrm{H}}-\mathrm{H}_{\mathrm{OG}}$ of $\mathrm{ChCl}-\mathrm{EG}$ at $298 \mathrm{~K}$. The distributions of $\mathrm{O}_{\mathrm{H}}-\mathrm{H}_{\mathrm{O}}$ and $\mathrm{O}_{\mathrm{HG}}-\mathrm{H}_{\mathrm{O}}$ are excluded from the plot due to their similarity with the $\mathrm{O}_{\mathrm{H}}-\mathrm{H}_{\mathrm{OG}}$ data. (The groups in grey on the structural formulae correspond to chemical functional groups, not to the atom types used in the simulation.)

We implemented the Tang-Toennies damping function, the periodic perturbation method and the temperaturegrouped Nosé-Hoover thermostat in the LAMMPS molecular dynamics code. Examples of the input files are available in code repositories. ${ }^{49}$

The main contribution of this work is the extension of the CL\&Pol force field to three classes of systems dominated by strong hydrogen bonds or containing small
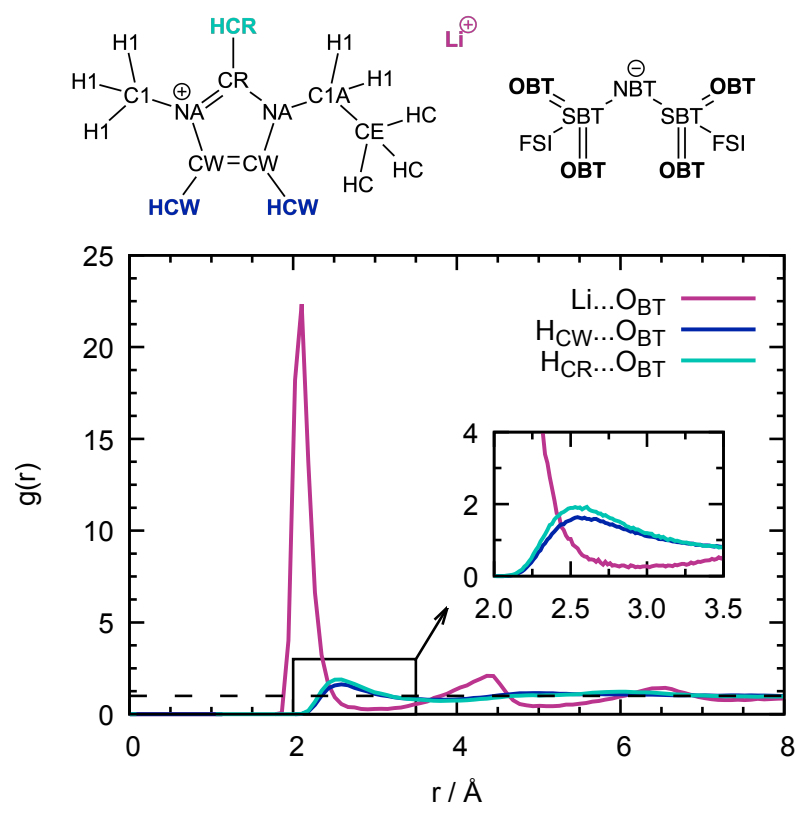

FIG. 8. Radial distribution function of $\mathrm{Li}-\mathrm{O}_{\mathrm{BT}}, \mathrm{H}_{\mathrm{CW}}-\mathrm{O}_{\mathrm{BT}}$ and $\mathrm{H}_{\mathrm{CR}}-\mathrm{O}_{\mathrm{BT}}$ in $\mathrm{Li}[\mathrm{FSI}]-\left[\mathrm{C}_{2} \mathrm{C}_{1} \mathrm{im}\right][\mathrm{FSI}]\left(x_{\mathrm{Li}[\mathrm{FSI}]}=0.2\right)$ at $338 \mathrm{~K}$.

ions: protic ionic liquids, deep eutectic solvents and alkali metal electrolytes. We provide here results on examples of the three classes, and a general and robust methodology has been proposed that can be readily extended to many similar systems, benefiting from the transferability of the fragment approach underlying the CL\&Pol force field. The result of our polarizable simulations contribute to the understanding of the nature of hydrogen-bonding in ethylammonium nitrate, an important protic ionic liquid and intensely studied solvent, for which two conflicting views have been proposed in the literature 


\section{ACKNOWLEDGMENTS}

This work was supported by an IDEX Lyon Fellowship (ANR-16-IDEX-005) and by ANR project LIQUI2D (ANR-18-CE09-0018). Computational tasks were performed using resources from GENCI-IDRIS (Grant 2019A0070800609) and on the Pôle Scientifique de Modélisation Numérique (PSMN) at ENS de Lyon.

\section{REFERENCES}

${ }^{1}$ T. L. Greaves and C. J. Drummond, "Protic ionic liquids: Properties and applications," Chem. Rev. 108, 206-237 (2008).

${ }^{2}$ T. L. Greaves and C. J. Drummond, "Protic ionic liquids: Evolving structure-property relationships and expanding applications," Chem. Rev. 115, 11379-11448 (2015).

${ }^{3}$ K. Fumino, A. Wulf, and R. Ludwig, "Hydrogen bonding in protic ionic liquids: Reminiscent of water," Angew. Chem. Int. Ed. 48, 3184-3186 (2009).

${ }^{4}$ R. Hayes, S. Imberti, G. G. Warr, and R. Atkin, "Amphiphilicity determines nanostructure in protic ionic liquids," Phys. Chem. Chem. Phys. 13, 3237-3247 (2011).

${ }^{5}$ R. Hayes, S. Imberti, G. G. Warr, and R. Atkin, Angew. Chem. Int. Ed. 52, 4623-4627 (2013).

${ }^{6}$ X. Song, H. Hamano, B. Minofar, R. Kanzaki, K. Fujii, Y. Kameda, S. Kohara, M. Watanabe, S.-i. Ishiguro, and Y. Umebayashi, "Structural heterogeneity and unique distorted hydrogen bonding in primary ammonium nitrate ionic liquids studied by high-energy x-ray diffraction experiments and md simulations," J. Phys. Chem. B 116, 2801-2813 (2012).

${ }^{7}$ O. Russina, A. Mariani, R. Caminiti, and A. Triolo, "Structure of a binary mixture of ethylammonium nitrate and methanol," J. Solution Chem. 44, 669-685 (2015).

${ }^{8}$ L. Gontrani, E. Bodo, A. Triolo, F. Leonelli, P. D'Angelo, V. Migliorati, and R. Caminiti, "The interpretation of diffraction patterns of two prototypical protic ionic liquids: a challenging task for classical molecular dynamics simulations," J. Phys. Chem. B 116, 13024-13032 (2012).

${ }^{9} \mathrm{~T}$. Zentel and O. Kühn, "Properties of hydrogen bonds in the protic ionic liquid ethylammonium nitrate," Theor. Chem. Acc. 136, 87 (2017).

${ }^{10} \mathrm{~S}$. Zahn, J. Thar, and B. Kirchner, "Structure and dynamics of the protic ionic liquid monomethylammonium nitrate $\left(\left[\mathrm{CH}_{3} \mathrm{NH}_{3}\right]\left[\mathrm{NO}_{3}\right]\right)$ from ab initio molecular dynamics simulations," J. Chem. Phys. 132, 124506 (2010).

${ }^{11}$ E. Bodo, A. Sferrazza, R. Caminiti, S. Mangialardo, and P. Postorino, "A prototypical ionic liquid explored by ab initio molecular dynamics and raman spectroscopy," J. Chem. Phys. 139 (2013), 10.1063/1.4823824.

${ }^{12}$ E. L. Smith, A. P. Abbott, and K. S. Ryder, "Deep eutectic solvents (dess) and their applications," Chem. Rev. 114, 1106011082 (2014).

${ }^{13}$ Q. Zhang, K. De Oliveira Vigier, S. Royer, and F. Jérôme, "Deep eutectic solvents: syntheses, properties and applications," Chem. Soc. Rev. 41, 7108-7146 (2012).

${ }^{14}$ A. Lewandowski and A. Świderska-Mocek, "Ionic liquids as electrolytes for li-ion batteries - an overview of electrochemical studies," J. Power Sources 194, 601-609 (2009).

${ }^{15}$ L. Suo, O. Borodin, T. Gao, M. Olguin, J. Ho, X. Fan, C. Luo, C. Wang, and K. Xu, "“water-in-salt"electrolyte enables high-voltage aqueous lithium-ion chemistries," Science 350, 938 (2015).

${ }^{16}$ H. Yoon, P. C. Howlett, A. S. Best, M. Forsyth, and D. R. MacFarlane, "Fast charge/discharge of li metal batteries using an ionic liquid electrolyte," J. Electrochem. Soc. 160, A1629-A1637 (2013).
${ }^{17}$ A. Massaro, J. Avila, K. Goloviznina, I. Rivalta, C. Gerbaldi, M. Pavone, M. F. Costa Gomes, and A. A. H. Padua, "Sodium diffusion in ionic liquid-based electrolytes for na-ion batteries: the effect of polarizable force fields," Phys. Chem. Chem. Phys. 22, 20114-20122 (2020).

${ }^{18}$ D. Bedrov, J.-P. Piquemal, O. Borodin, A. D. Mackerell, B. Roux, and C. Schröder, "Molecular Dynamics Simulations of Ionic Liquids and Electrolytes Using Polarizable Force Fields," Chem. Rev. 119, 7940-7995 (2019).

${ }^{19}$ S. Y. Noskov, G. Lamoureux, and B. Roux, "Molecular dynamics study of hydration in ethanol-water mixtures using a polarizable force field," J. Phys. Chem. B 109, 6705-6713 (2005).

${ }^{20}$ O. Borodin, "Polarizable force field development and molecular dynamics simulations of ionic liquids," J. Phys. Chem. B 113, 11463-11478 (2009).

${ }^{21}$ J. W. Ponder, C. Wu, P. Ren, V. S. Pande, J. D. Chodera, M. J. Schnieders, I. Haque, D. L. Mobley, D. S. Lambrecht, R. A. DiStasio, M. Head-Gordon, G. N. I. Clark, M. E. Johnson, and T. Head-Gordon, "Current status of the amoeba polarizable force field," J. Phys. Chem. B 114, 2549-2564 (2010).

${ }^{22}$ M. C. C. Ribeiro, "Polarization effects in molecular dynamics simulations of glass-formers $\mathrm{Ca}\left(\mathrm{NO}_{3}\right)_{2} \cdot \mathrm{nH}_{2} \mathrm{O}, n=4,6$ and 8 ," The Journal of Chemical Physics 132, 134512 (2010).

${ }^{23}$ H. Yu, T. W. Whitfield, E. Harder, G. Lamoureux, I. Vorobyov, V. M. Anisimov, A. D. MacKerell, and B. Roux, "Simulating monovalent and divalent ions in aqueous solution using a drude polarizable force field," J. Chem. Theory Comput. 6, 774-786 (2010).

24 J. Chowdhary, E. Harder, P. E. M. Lopes, L. Huang, A. D. MacKerell, and B. Roux, "A polarizable force field of dipalmitoylphosphatidylcholine based on the classical drude model for molecular dynamics simulations of lipids," J. Phys. Chem. B 117, 91429160 (2013).

${ }^{25}$ K. Goloviznina, J. N. Canongia Lopes, M. Costa Gomes, and A. A. H. Pádua, "Transferable, Polarizable Force Field for Ionic Liquids," J. Chem. Theory Comput. 15, 5858-5871 (2019).

${ }^{26}$ A. A. H. Pádua, "Resolving Dispersion and Induction Components for Polarisable Molecular Simulations of Ionic Liquids," J. Chem. Phys. 146, 204501 (2017), 1703.01540.

${ }^{27}$ J. Huang, P. E. M. Lopes, B. Roux, and A. D. MacKerell, "Recent Advances in Polarizable Force Fields for Macromolecules: Microsecond Simulations of Proteins Using the Classical Drude Oscillator Model," J. Phys. Chem. Lett. 5, 3144-3150 (2014).

${ }^{28}$ J. A. Lemkul, J. Huang, B. Roux, and A. D. MacKerell, "An Empirical Polarizable Force Field Based on the Classical Drude Oscillator Model: Development History and Recent Applications," Chem. Rev. 116, 4983-5013 (2016).

${ }^{29}$ J. N. Canongia Lopes, J. Deschamps, and A. A. H. Pádua, "Modeling Ionic Liquids Using a Systematic All-Atom Force Field," J. Phys. Chem. B 108, 2038-2047 (2004).

${ }^{30}$ J. N. Canongia Lopes and A. A. H. Padua, "CL\&P: A generic and systematic force field for ionic liquids modeling," Theor. Chem. Acc. 131, 1129 (2012).

${ }^{31}$ A. A. H. Padua, "github.com/agiliopadua/ilff," (2012).

${ }^{32}$ B. T. Thole, "Molecular polarizabilities calculated with a modified dipole interaction," Chem. Phys. 59, 341-350 (1981).

${ }^{33}$ T. Taylor, M. Schmollngruber, C. Schröder, and O. Steinhauser, "The effect of thole functions on the simulation of ionic liquids with point induced dipoles at various densities," J. Chem. Phys. 138, 204119 (2013).

${ }^{34}$ J. A. Lemkul, B. Roux, D. van der Spoel, and A. D. MacKerell Jr., "Implementation of extended Lagrangian dynamics in GROMACS for polarizable simulations using the classical Drude oscillator model," J. Comp. Chem. 36, 1473-1479 (2015).

${ }^{35}$ J. Huang, J. A. Lemkul, P. K. Eastman, and A. D. MacKerell Jr., "Molecular dynamics simulations using the drude polarizable force field on GPUs with OpenMM: Implementation, validation, and benchmarks," J. Comput. Chem. 39, 1682-1689 (2018).

${ }^{36}$ B. R. Brooks, R. E. Bruccoleri, B. D. Olafson, D. J. States, 
S. Swaminathan, and M. Karplus, "Charmm: A program for macromolecular energy, minimization, and dynamics calculations," J. Comp. Chem. 4, 187-217 (1983).

${ }^{37}$ A.-P. E. Kunz and W. F. van Gunsteren, "Development of a nonlinear classical polarization model for liquid water and aqueous solutions: Cos/d," J. Phys. Chem. A 113, 11570-11579 (2009).

${ }^{38} \mathrm{~K}$. T. Tang and J. Peter Toennies, "An improved simple model for the van der Waals potential based on universal damping functions for the dispersion coefficients," J. Chem. Phys. 80, 3726-3741 (1984).

${ }^{39} \mathrm{M}$. Wilson and P. A. Madden, "Polarization effects in ionic systems from first principles," J. Phys. Condens. Matter 5, 26872706 (1993).

${ }^{40}$ P. Jemmer, M. Wilson, P. A. Madden, and P. W. Fowler, "Dipole and quadrupole polarization in ionic systems: Ab initio studies," J. Chem. Phys. 111, 2038-2049 (1999).

${ }^{41} \mathrm{M}$. Salanne and P. A. Madden, "Polarization effects in ionic solids and melts," Mol. Phys. 109, 2299-2315 (2011).

${ }^{42}$ M. Salanne, L. J. A. Siqueira, A. P. Seitsonen, P. A. Madden, and B. Kirchner, "From molten salts to room temperature ionic liquids: Simulation studies on chloroaluminate systems," Faraday Discuss. 154, 171-188 (2012).

${ }^{43}$ S. Sharma, M. S. Emerson, F. Wu, H. Wang, E. J. Maginn, and C. J. Margulis, "Sem-drude model for the accurate and efficient simulation of mgcl2-kcl mixtures in the condensed phase," J. Phys. Chem. A 124, 7832-7842 (2020).

${ }^{44}$ W. L. Jorgensen, D. S. Maxwell, and J. Tirado-Rives, "Development and Testing of the OPLS All-Atom Force Field on Conformational Energetics and Properties of Organic Liquids," J. Am. Chem. Soc. 118, 11225-11236 (1996).

${ }^{45}$ S. J. Plimpton, "Fast Parallel Algorithms for Short-Range Molecular Dynamics," J. Comput. Phys. 117, 1-19 (1995).

${ }^{46}$ A. Dequidt, J. Devémy, and A. A. H. Padua, "Thermalized Drude Oscillators with the LAMMPS Molecular Dynamics Simulator," J. Chem. Info. Model. 56, 260-268 (2016).

${ }^{47}$ A. A. H. Padua, "github.com/agiliopadua/fftool," (2013).

${ }^{48}$ L. Martínez, R. Andrade, E. G. Birgin, and J. M. Martínez, "PACKMOL: A package for building initial configurations for molecular dynamics simulations," J. Comp. Chem. 30, 2157-2164 (2009)

${ }^{49}$ A. A. H. Padua, "github.com/agiliopadua/pol il," (2019).

${ }^{50}$ C. Y. Son, J. G. McDaniel, Q. Cui, and A. Yethiraj, "Proper Thermal Equilibration of Simulations with Drude Polarizable Models: Temperature-Grouped Dual-Nosé-Hoover Thermostat," J. Phys. Chem. Lett. 10, 7523-7530 (2019).

${ }^{51} \mathrm{M}$. Brehm and B. Kirchner, "Travis - a free analyzer and visualizer for monte carlo and molecular dynamics trajectories," J. Chem. Inf. Model. 51, 2007-2023 (2011).

${ }^{52}$ O. Hollóczki, M. Macchiagodena, H. Weber, M. Thomas, M. Brehm, A. Stark, O. Russina, A. Triolo, and B. Kirchner, "Triphilic ionic-liquid mixtures: Fluorinated and non-fluorinated aprotic ionic-liquid mixtures," ChemPhysChem 16, 3325-3333 (2015).

${ }^{53}$ E. Maginn, R. Messerly, D. Carlson, D. Roe, and J. Elliott, "Best Practices for Computing Transport Properties 1. SelfDiffusivity and Viscosity from Equilibrium Molecular Dynamics [Article v1.0]," Living J. Comp. Mol. Sci. 1 (2018), 10.33011/livecoms.1.1.6324.

${ }^{54} \mathrm{~B}$. Hess, "Determining the shear viscosity of model liquids from molecular dynamics simulations," J. Chem. Phys. 116, 209-217 (2001).

${ }^{55}$ I.-C. Yeh and G. Hummer, "System-size dependence of diffusion coefficients and viscosities from molecular dynamics simulations with periodic boundary conditions," J. Phys. Chem. B 108, 15873-15879 (2004).

${ }^{56} \mathrm{G}$. Lamoureux and B. Roux, "Modeling induced polarization with classical Drude oscillators: Theory and molecular dynamics simulation algorithm," J. Chem. Phys. 119, 3025-3039 (2003).

${ }^{57}$ M. J. Frisch, G. W. Trucks, H. B. Schlegel, G. E. Scuseria, M. A. Robb, J. R. Cheeseman, G. Scalmani, V. Barone, G. A. Peters- son, H. Nakatsuji, X. Li, M. Caricato, A. V. Marenich, J. Bloino, B. G. Janesko, R. Gomperts, B. Mennucci, H. P. Hratchian, J. V. Ortiz, A. F. Izmaylov, J. L. Sonnenberg, D. WilliamsYoung, F. Ding, F. Lipparini, F. Egidi, J. Goings, B. Peng, A. Petrone, T. Henderson, D. Ranasinghe, V. G. Zakrzewski, J. Gao, N. Rega, G. Zheng, W. Liang, M. Hada, M. Ehara, K. Toyota, R. Fukuda, J. Hasegawa, M. Ishida, T. Nakajima, Y. Honda, O. Kitao, H. Nakai, T. Vreven, K. Throssell, J. A. Montgomery, Jr., J. E. Peralta, F. Ogliaro, M. J. Bearpark, J. J. Heyd, E. N. Brothers, K. N. Kudin, V. N. Staroverov, T. A. Keith, R. Kobayashi, J. Normand, K. Raghavachari, A. P. Rendell, J. C. Burant, S. S. Iyengar, J. Tomasi, M. Cossi, J. M. Millam, M. Klene, C. Adamo, R. Cammi, J. W. Ochterski, R. L. Martin, K. Morokuma, O. Farkas, J. B. Foresman, and D. J. Fox, "Gaussian 16 Revision B.01," (2016), gaussian Inc. Wallingford CT.

${ }^{58}$ C. M. Breneman and K. B. Wiberg, "Determining atom-centered monopoles from molecular electrostatic potentials. The need for high sampling density in formamide conformational analysis," J. Comput. Chem. 11, 361-373 (1990).

${ }^{59}$ E. Heid, A. Szabadi, and C. Schröder, "Quantum mechanical determination of atomic polarizabilities of ionic liquids," Phys. Chem. Chem. Phys. 20, 10992-10996 (2018).

${ }^{60}$ E. Heid, M. Heindl, P. Dienstl, and C. Schröder, "Additive polarizabilities of halides in ionic liquids and organic solvents," The Journal of Chemical Physics 149, 044302 (2018).

${ }^{61}$ D. Bohne, S. Fischer, and E. Obermeier, "Thermal, conductivity, density, viscosity, and prandtl-numbers of ethylene glycol-water mixtures," Ber. Bunsenges. Phys. Chem. 88, 739-742 (1984).

${ }^{62}$ N. Chandrasekhar and P. Krebs, "The spectra and the relative yield of solvated electrons produced by resonant photodetachment of iodide anion in ethylene glycol in the temperature range $296 \leq t \leq 453$ K," J. Chem. Phys. 112, 5910-5914 (2000).

${ }^{63}$ P. A. Hunt, C. R. Ashworth, and R. P. Matthews, "Hydrogen bonding in ionic liquids," Chemical Society Reviews 44, 12571288 (2015).

${ }^{64}$ A. Mariani, M. Bonomo, B. Wu, B. Centrella, D. Dini, E. W. Castner, and L. Gontrani, "Intriguing transport dynamics of ethylammonium nitrate-acetonitrile binary mixtures arising from nano-inhomogeneity," Phys. Chem. Chem. Phys. 19, 2721227220 (2017)

${ }^{65}$ C. D'Agostino, R. C. Harris, A. P. Abbott, L. F. Gladden, and M. D. Mantle, "Molecular motion and ion diffusion in choline chloride based deep eutectic solvents studied by $1 \mathrm{~h}$ pulsed field gradient nmr spectroscopy," Physical Chemistry Chemical Physics 13, 21383-21391 (2011).

${ }^{66} \mathrm{H}$. Matsumoto, H. Sakaebe, and K. Tatsumi, "Preparation of room temperature ionic liquids based on aliphatic onium cations and asymmetric amide anions and their electrochemical properties as a lithium battery electrolyte," J. Power Sources 146, 45-50 (2005).

${ }^{67}$ P. Judeinstein, C. Iojoiu, J.-Y. Sanchez, and B. Ancian, "Proton conducting ionic liquid organization as probed by nmr: Selfdiffusion coefficients and heteronuclear correlations," J. Phys. Chem. B 112, 3680-3683 (2008).

${ }^{68}$ W. A. Henderson, P. Fylstra, H. C. De Long, P. C. Trulove, and S. Parsons, "Crystal structure of the ionic liquid etnh3no3insights into the thermal phase behavior of protic ionic liquids," Phys. Chem. Chem. Phys. 14, 16041-16046 (2012).

${ }^{69}$ Y. Umebayashi, W.-L. Chung, T. Mitsugi, S. Fukuda, M. Takeuchi, K. Fujii, T. Takamuku, R. Kanzaki, and S.-i. Ishiguro, "Liquid structure and the ion-ion interactions of ethylammonium nitrate ionic liquid studied by large angle x-ray scattering and molecular dynamics simulations," J. Comput. Chem., Jpn. 7, 125-134 (2008).

${ }^{70}$ T. L. Greaves, D. F. Kennedy, S. T. Mudie, and C. J. Drummond, "Diversity observed in the nanostructure of protic ionic liquids," J. Phys. Chem. B 114, 10022-10031 (2010).

${ }^{71}$ R. Atkin and G. G. Warr, "The smallest amphiphiles: Nanostructure in protic room-temperature ionic liquids with short alkyl 
groups," J. Phys. Chem. B 112, 4164-4166 (2008).

${ }^{72}$ V. Alizadeh, F. Malberg, A. A. H. Pádua, and B. Kirchner, "Are there magic compositions in deep eutectic solvents? effects of composition and water content in choline chloride/ethylene glycol from ab initio molecular dynamics," J. Phys. Chem. B 124, 74337443 (2020).

${ }^{73}$ E. Bolimowska, F. Castiglione, J. Devemy, H. Rouault, A. Mele, A. A. H. Pádua, and C. C. Santini, "Investigation of li+ cation coordination and transportation, by molecular modeling and $\mathrm{nmr}$ studies, in a lintf2-doped ionic liquid-vinylene carbonate mixture," J. Phys. Chem. B 122, 8560-8569 (2018).

${ }^{74}$ K. Matsumoto, E. Nishiwaki, T. Hosokawa, S. Tawa, T. Nohira, and R. Hagiwara, "Thermal, physical, and electrochemical properties of $\mathrm{Li}\left[\mathrm{N}\left(\mathrm{SO}_{2} \mathrm{~F}\right)_{2}\right]$-[1-ethyl-3-methylimidazolium] $\left[\mathrm{N}\left(\mathrm{SO}_{2} \mathrm{~F}\right)_{2}\right]$ ionic liquid electrolytes for li secondary batteries operated at room and intermediate temperatures," J. Phys. Chem. C 121, 9209-9219 (2017).

${ }^{75}$ L. Zhao, T. Cheng, and H. Sun, "On the accuracy of predicting shear viscosity of molecular liquids using the periodic perturbation method," J. Chem. Phys. 129, 144501 (2008).

${ }^{76} \mathrm{Z}$. Gong and H. Sun, "Extension of team force-field database to ionic liquids," J. Chem. Eng. 64, 3718-3730 (2019).

${ }^{77}$ Z. Gong and H. Sun, "Pressure-viscosity relation of 2,2,4trimethylhexane predicted using all-atom team force field," Fluid Phase Equilibr. 497, 64-70 (2019).

\section{Appendix A: LAMMPS implementation}

The Tang-Toennies damping function is implemented as a coul/tt pair style

pair_style hybrid/overlay \
$1 \mathrm{j} /$ cut/thole/long $2.612 .0 \mathrm{coul} / \mathrm{tt} 412.0$

where the degree of polynomial is 4 and the cutoff is $12 \AA$. The pair_coeff section of the input file requires the $b$ parameter and the pre-exponential factor (set to 1.0 in the present work) for the interactions between the highly-charged atom and all Drude dipoles.

$$
\text { pair_coeff } 12 \text { coul/tt } 4.51 .0
$$

This new pair style is available in the USER-DRUDE package of LAMMPS, version 29Oct2020. A detailed description of input files preparation for strongly H-bonded systems and the usage of the coul/tt pair style is provided in the code repositories. ${ }^{49}$

The tgNH thermostat is implemented as a fix command in LAMMPS. In our implementation, the degrees of freedom (DOF) of the system are separated into three contributions: the motion of molecular center of mass (MCOM), the motion of center of mass of atom-Drude pairs (COM) relative to the MCOM, and the relative motion between atom-Drude pairs. The tgNH thermostat is composed of three independent thermostats applied to these three categories of DOF. The following example thermalizes the motions of MCOM and COM at $298 \mathrm{~K}$ with a time constant of $100 \mathrm{fs}$, and the relative motion of Drude pairs at $1 \mathrm{~K}$ with a time constant of $20 \mathrm{fs}$. This fix command also calculates the temperatures of these three DOF, which can be output by the themro_style command.

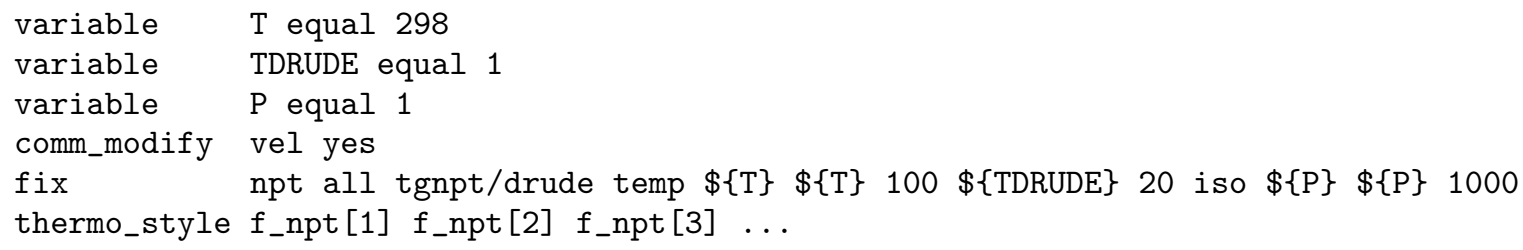

The periodic perturbation method is implemented as a combination of $\mathrm{fix}$ and compute commands, which have been included in LAMMPS, version 5May2020. In the example below, the reciprocal viscosity is calculates with $\operatorname{tg} \mathrm{NH}$ thermostat and an acceleration strength of $0.02 \mathrm{~nm} \mathrm{ps}^{-2}$. The cosine-shaped acceleration in the $x$ direction with periodicity in the $z$ direction is applied by using the fix accelerate/cos command. The compute viscosity/cos command calculates the velocity profile in response to the acceleration. Then, the reciprocal vis- cosity is calculated from the velocity profile and can be outputted by a thermo_style command. The generated collective velocities along $x$ should not be taken into account by thermostat. Therefore, the fix_modify command should be invoked to remove and restore the velocity profile before and after thermostating. This method is compatible with both the tgNH thermostat and traditional Nose-Hoover thermostat. For non-polarizable simulation, simply replace the fix tgnpt/drude command with a corresponding fix npt command.

$\begin{array}{ll}\text { variable } & \text { A equal } 0.02 e-5 \# \text { angstrom/fs^2 } \\ \text { fix } & \text { cos all accelerate/cos } \$\{A\} \\ \text { compute } & \text { cos all viscosity/cos } \\ \text { variable } & \text { density equal density } \\ \text { variable } & \text { lz equal lz } \\ \text { variable } & \text { vMax equal c_cos }[7]\end{array}$




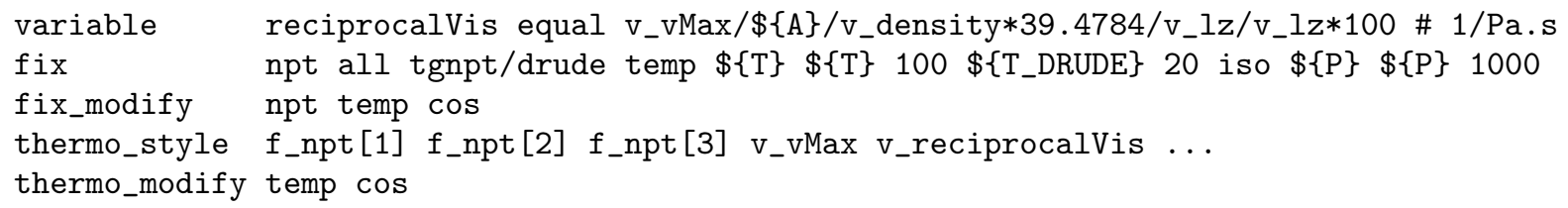

Appendix B: Choice of acceleration strength in the periodic perturbation method for viscosity evaluation

We calculated viscosity using the periodic perturbation method with acceleration strengths in the range $0.005-$ $0.1 \times 10^{-5} \AA \mathrm{fs}^{-2}$. As discussed previously by Hess, ${ }^{54}$ too big $\mathcal{A}$ values lead to high share rates resulting in viscosity underestimation. On the other hand, too small $\mathcal{A}$ values introduce noise in the calculated data, as reported in the Table VII. One could suggest to extrapolate the viscosity to zero acceleration strength, ${ }^{75-77}$ but the calculation at a series of $\mathcal{A}$ value has high computational cost when a polarizable force field is used. Thus, we propose to evaluate viscosity at one value of acceleration strength, $0.02 \times 10^{-5} \AA \mathrm{fs}^{-2}$, found to be optimal for the systems discussed in this paper.

TABLE VII. Viscosity values of EG and EAN at $298 \mathrm{~K}$ at different acceleration strengths, obtained using the periodic perturbation method.

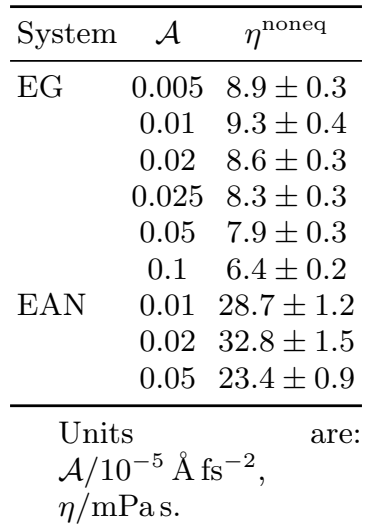

